\title{
Number Plate Recognition (ANR) using Crimmins Complementary Hulling Algorithm
}

\author{
Kiran Sonavane \\ Application Engineer \\ Assam, India
}

\begin{abstract}
The crime of stolen vehicle in the metro city has rise now a days, to track the stolen vehicle the cops required the help of automatic Number Plate recognition (ANR). The use of ANR are not limited to the track stolen car but many more like automatic car parking system, automatic toll collection system etc. but the ANR system has many issues in implementation. Some ANR uses hardware repository to track the number of the vehicle which uses the CCTV camera to capture vehicle image. the extracting the number from the vehicle image. the image has to be noise free. In proposed method I implemented the new approach to remove the noise from vehicle image which is generated by the camera due to low sunlight as a result image will contain salt and paper noise. In proposed method crimmins speckle algorithm is used to remove the salt and paper noise.
\end{abstract}

\section{Keywords}

NN(Neural Network), OCR, preprocessing, ANR (Automatic Number plate Recognition system), crimmins speckle.

\section{INTRODUCTION}

The ANR (automatic number plate recognition) is plays a vital role in the metropolitan city's traffic monitoring and parking system in the mall, cinemas and restaurants and many more.ANR helps to the cops to track the theft and robbers during the trapping of robbers. In ANR system the recognition of the number plate is still challenging task because of the poor illumination condition, poor sunlight, etc[13]. If the camera is not high resolution or high pixel then it will produce the bad image or image with noise. Many of the time due to the low sunlight the image may contain the salt and paper noise. To remove the noise from such a vehicle image I have applied the crimmins speckle algorithm to remove the noise from vehicle image. the ANR system consist of following steps to recognize the number from vehicle image.

1. Image Capture: In this step image is captured either from camera or from video (one frame of the video).camera also plays an important role in ANR. If the resolution is higher the recognition rate will be higher.

2. Image Preprocessing: If the image captured by the camera is not good or contains some noise then in preprocessing step all the noise can be removed.

3. ROI(region of interest): In this step the Number plate area from the vehicle image is extracted out. for extracting the number plate region many techniques has been used like feature extraction. Region based extraction.

4. OCR(Optical Character Recognition): In this step the extracted number plate region processed to recognize the character in the image. there are many methods are there such as template matching. Support vector machine, Neural Network etc.
The Proposed method is the categorized in image Preprocessing.

\section{LITRATURE REVIEW}

Every year many researchers came with new and better algorithm for ANR. In [9] they have implemented the ANR system for Indian vehicle where number plate is written in two rows. The advantage of their system is the efficiency which is $82 \%$. They have implemented OCR (optical character recognition) using template matching.

The ANR System is also implemented on android devises like phone, tablets and android laptops etc. In [11] abdul and teddy have implemented the number plate recognition for android system. The image is captured from the android phone camera. The phone camera might have the poor resolution which leads to apply the additional filters to filter the image. In [11] they have preprocessed the image using contrast enhancement, filtering and stretching.OCR is implemented using Neural Network.

ROI can be extracted by using advanced algorithm like GA (genetic algorithm) [10] [12] [14]. In [14] they have used the GA for locating the license plate and selecting the optimum License Plate symbol locations depending on the input geometric relationship matrix (GRM) that defines the geometrical relationships betIen the symbols in the concerned License plate.

\section{PROPOSED METHOD}

In the ANR System the image acquisition is important stage where image in being captured from the CCTV camera and processed in the system itself and extract the number from the image and stored the extracted character in the text format in the database for future use. These database can be further used by the cops or any civil department to track the traffic and robbed cars in the city. But if the acquired image is not properly acquired then it will give the error in the OCR (Optical character recognition) stage. The error may be the wrong text in the output the OCR stage may predict the word ' $\mathrm{O}$ ' instead of Number ' 0 ' or wise versa. In proposed system I identified the possible errors because of the incomplete image acquisition stage also I eliminated the salt and paper noise present in the car image after image captured. the flow chart of the proposed system is shown in below Fig 1

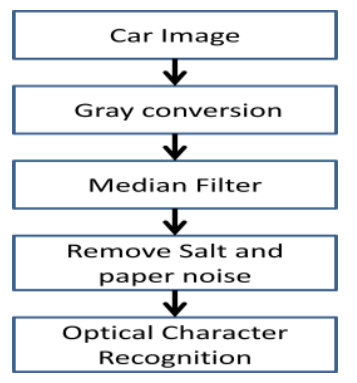

Fig 1: Flowchart of the Proposed system. 
Following are the stages in the proposed system

\section{Image Acquisition or Preprocessing}

\section{Extract the Region of Interest (ROI)}

\section{Optical Character Recognition}

\subsection{Preprocessing}

Input image is first convert to gray level based on threshold level of the pixel. But sometimes the in low light environment image captured by the camera or CCTV camera is not cleared it has some effects which is similar to salt and paper noise in the image[2]. it is because of low shutter speed, higher gain or both[2].so to remove the salt and paper noise from the image only median filter is not sufficient to remove the noise. In order to remove the noise from such image I need on additional filter. In these paper I have used crimmins speckle removal algorithm to remove the effect of salt and paper noise.

This algorithm provides the better result than median filter[1]. It reduces the intensity of salt and paper noise from the image. and also blurs cthe high frequency components from the images. The blur part of the image later being sharpen by the sharpening filter.

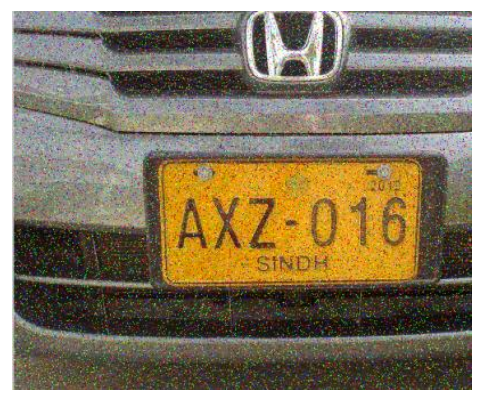

(a)

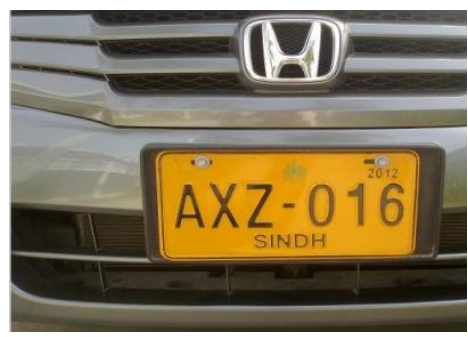

(b)

Fig 2: (a) Before applying the crimmins speckle algorithm.

\section{(b) After applying the crimmins speckle algorithm.}

In the Fig.2 (a) shows the CCTV camera image taken at low light environment due to low light the image contained salt and paper noise in the image. to remove this effect applied the the crimmins speckle algorithm which remove the all the noise efficiently with less amount of time.

\subsection{Cummins speckle algorithm: In these} algorithm the pixel value of each pixel is capered with the pixel value of its surroundings and update the that pixel values. Updating the values has following rules

The flow chart of algorithm is shown in below Fig. 3 In the fig. 3 ' $\mathrm{X}$ ' is any pixel in an image which contains noise. And
' $\mathrm{v}(\mathrm{x})$ ' is the value of that pixel.

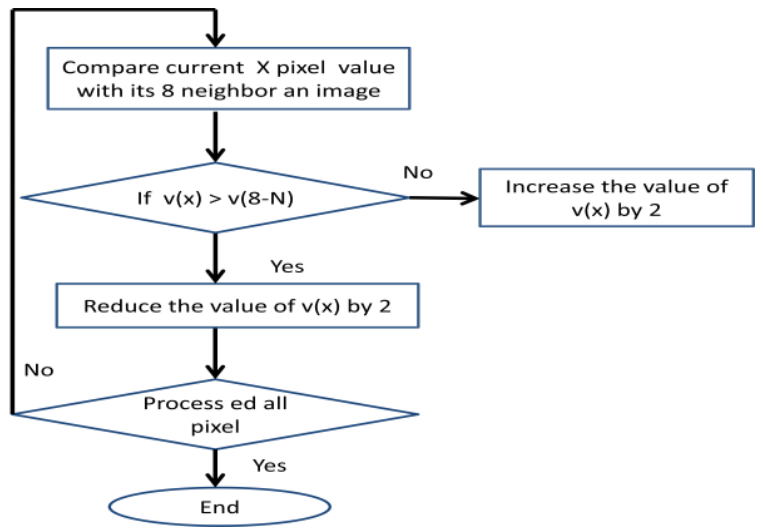

Fig 3: Flowchart of Cummins speckle algorithm.

\subsection{ROI (region of interest)}

Once the preprocessing is done next step is to extract the number plate region from the processed image. in these paper I have implemented the ROI based on the possible position of the number plate in the image. Usually, the number plate is always being at the loIr part of the image [5]. It is due to the position of the camera in the toll system or in traffic monitoring systems always fixed and takes car images in periodic manner. I have used the loIr co-ordinates of the image to extract out the rectangular number plate region. The output of the extracted number plate is shown in the Fig:3 as below.

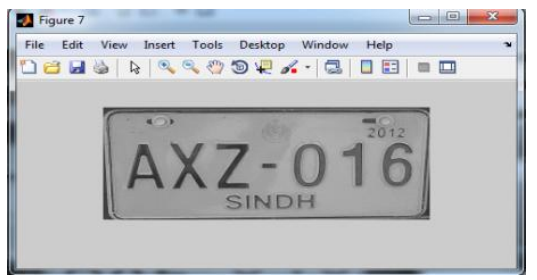

Fig 4: Extracted Number Plate output.

\subsection{OCR (Optical Character Recognition)}

After number plate extracted next step is optical character recognition. The accuracy of the ANR system is depends on how accurately the OCR can recognize the character from the extracted image. there are many available methods to implement the OCR. There are many methods are there to recognize the character from the image like Neural network, Genetic algorithms but all these methods are time consuming. Training the system in $\mathrm{NN}$ takes too much time[7]. And genetic algorithm requires too much computation time to compute the possible solution from the available solution using cross over, mutation method [6] [8]. In proposed system I have used the OCR based on the template matching [3] [4]. The extracted number plate first segmented out into number of character then each character is them compared with the template available in the database. Fig 4(a) shows the each separated extracted character from the number plate. If the extracted character matched with the database character then it returns the output in the text format. The output of the OCR step is shown below Fig 4(b). 


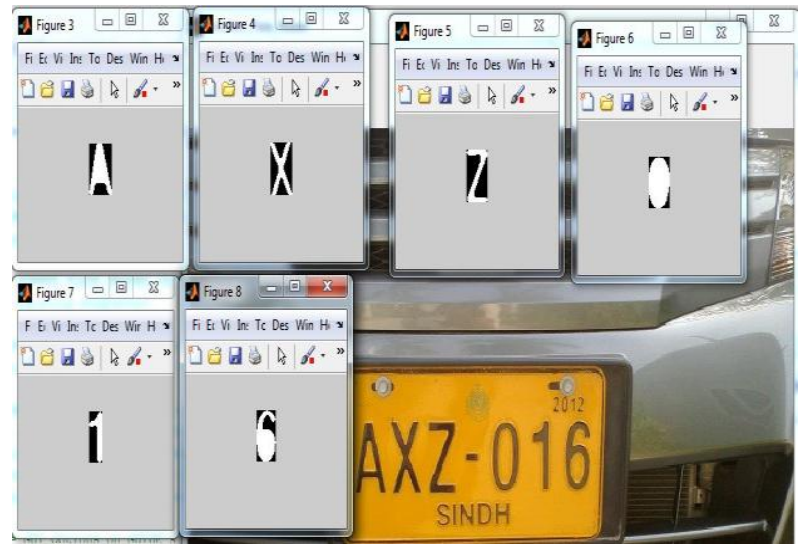

Fig 4(a): Individual Extracted characters.

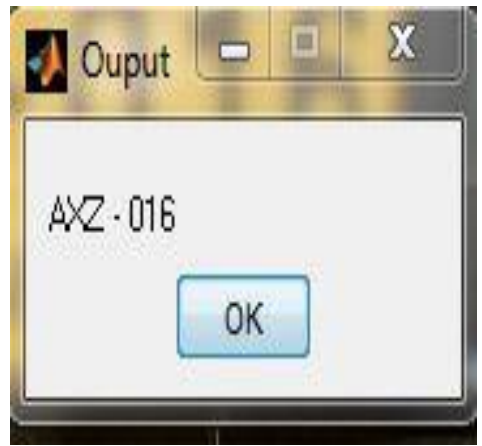

Fig 4(b): OCR output

\section{RESULTS}

I have tested the proposed algorithm for 60 different images taken by same camera and from same position. The tested images has been taken from poor light areas. And analyzes the algorithm result. Below Figure shoes the result of the proposed system is shown in below.

Table 1: Result of proposed system

\begin{tabular}{|l|l|l|l|}
\hline Stages of ANR & $\begin{array}{l}\text { Number } \\
\text { of images }\end{array}$ & $\begin{array}{l}\text { No. of } \\
\text { Correctly } \\
\text { detected } \\
\text { number } \\
\text { plate }\end{array}$ & $\begin{array}{l}\text { No. of } \\
\text { incorrectly } \\
\text { detected } \\
\text { number } \\
\text { plate }\end{array}$ \\
\hline Preprocessing & 60 & 58 & 2 \\
\hline $\begin{array}{l}\text { Extraction of } \\
\text { Number }\end{array}$ & 60 & 54 & 6 \\
\hline OCR & 60 & 52 & 8 \\
\hline
\end{tabular}

From above Table 1 only 2 images has not been correctly detected. So, the efficiency of the proposed algorithm is $98 \%$.

\section{CONCLUSION}

To remove the salt and paper noise effect from the image I applied the crimmins complementary hulling algorithm[1], but in order to get the good result the iteration has to increase which leads the performance degradation but for practical purpose these algorithm removes the salt and paper noise optimally.
During experiment phase, it is found that as the speckle level are increased in the image, the time required to remove the speckles are also increased. In the future work of the proposed system I will work to reduce the processing time. It can be done by using parallel programming like OpenMP. In order to achieve that goal just divide the loop iterations amongst the number of thread available which may reduce the processing time.

\section{REFERENCES}

[1] Ayoubi, R. ; Bayoumi, M. ; Ayoubi, R. "Real-time Parallelized Hybrid Median Filter for speckle removal in ultrasound images" Signal and Information Processing (GlobalSIP), 2014 IEEE Global Conference on Dec 2014.

[2] Arulmozhi K, Arumuga Perumal S, Jeevanmol Joy, Krishan Nallaperumal," Removal of Speckle and Impulse Noise from Indian License Plates using a 2Stage Median Filter", 2012 IEEE International Conference on Computational Intelligence and Computing Research.

[3] Priyanto Hidayatullah, Nurjannah Syakrani, Ida Suhartini, Wildan Muhlis, "Optical Character Recognition Improvement for License Plate Recognition in Indonesia",2012 UKSim-AMSS 6th European Modelling Symposium. Template matching ocr

[4] Kavita Ahuja, Preeti Tuli, "Object Recognition by Template Matching Using", International Journal of Advanced Research in Computer and Communication Engineering Vol. 2, Issue 3, March 2013.template matching

[5] Ghazal, M. Hajjdiab, H.,"License plate automatic detection and recognition using level sets and neural networks", 2013 1st International Conference on Communications, Signal Processing, and their Applications (ICCSPA).

[6] Samira Nigrel, Akash Ashokan, Bidisha Barua, Slesha Patil,Vaibhavi Patil," Identification of Vehicle by Number Plate Recognition Using Genetic Algorithm", International Journal of Advanced Research in Computer and Communication Engineering Vol. 4, Issue 2, February 2015

[7] Hung-Pin Chen, Ming-Hwa Chan,Yn-Her Juang ,' Recognition of blurred plate numbers using a novel algorithm based on Hopfield neural network", Systems, Man and Cybernetics, 2004 IEEE International Conference on (Volume:5 )

[8] Abo Samra and F. Khalefah,"Localization of License Plate Number Using Dynamic Image Processing Techniques and Genetic Algorithms" IEEE TRANSACTIONS ON EVOLUTIONARY COMPUTATION, VOL. 18, NO. 2, APRIL 2014

[9] Prathamesh Kulkarni (Student Member, IEEE), Ashish Khatri, Prateek Banga, Kushal Shah," Automatic Number Plate Recognition (ANPR) System for Indian conditions", 978-1-4244-3538-8/09/\$25.00 (02009 IEEE

[10] Kotaro HANEDA, Hiroshi HANAIZUMI, "A Flexible Method for Recognizing Four-Digit Numbers on A License-Plate in A Video Scene", ICIT 2012, 978-14673-0342-2112/\$31.00 @20 12 IEEE 
[11] Abdul Mutholib, Teddy Surya Gunawan," Design and Implementation of Automatic Number Plate Recognition on Android Platform", International Conference on Computer and Communication Engineering (ICCCE 2012), 3-5 July 2012, Kuala Lumpur, Malaysia 978-14673-0479-5/12/\$31.00 @2012 IEEE.

[12] P.Vijayalakshmi., M.Sumathi.," Design of Algorithm for Vehicle Identification by Number Plate Recognition", IEEE- Fourth International Conference on Advanced Computing, ICoAC 2012.
[13] Kiran Sonavane, Badal Soni and Umakanta Majhi. Article: Survey on Automatic Number Plate Recognition (ANR). International Journal of Computer Applications 125(6):1-4, September 2015. Published by Foundation of Computer Science (FCS), NY, USA.

[14] Abo Samra and F. Khalefah,'Localization of License Plate Number Using Dynamic Image Processing Techniques and Genetic Algorithms" IEEE TRANSACTIONS ON EVOLUTIONARY COMPUTATION, VOL. 18, NO. 2, APRIL 2014 